تأثير الكثافة النباتية على نمو وإنتاجية ثلاثة أصناف من محصول الشعير(Hordeum vulgare L)

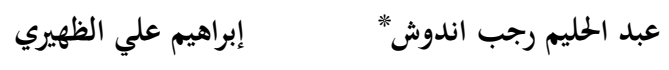

قسم الخحاصيل - كلية الزراعة - جامعة بنغازي

*abdulhalim.inooush@uob.edu.ly

نفذت بحربة حقلية بمنطقة بئر أرجام شرق سلوق خلال الموسم الزراعي 2019/2018 همدف معرفة تأثير الكثافات النباتية المناسبة على نمو

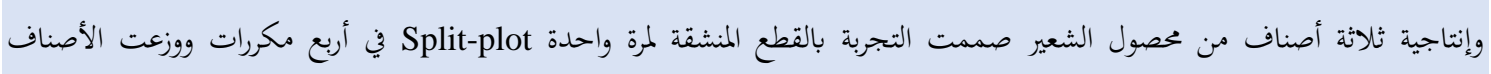

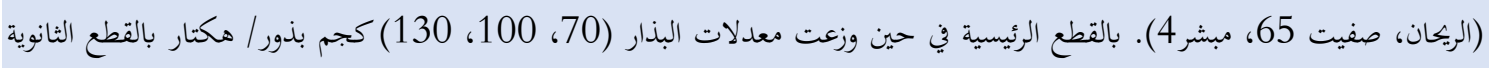
وكانت مساحة القطعة التجريبة 8م²، جميع العمليات الزراعية نفذت طبقاً للمتبع في المنطقة لإنتاج محصول الشعير وفق نظام الري التكميلي

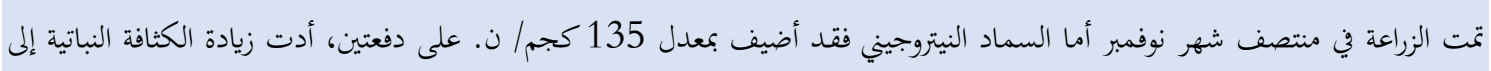

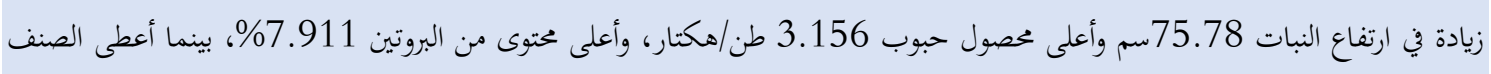

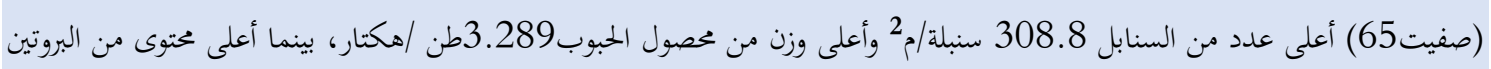

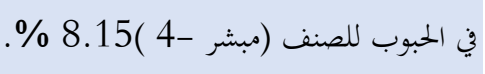

$$
\text { الكلمات المفتاحية: الأصناف - الشعير - الكثافات النباتية - النمو - الإنتاجية - سلوق. }
$$

1

يعد الشعير من محاصيل الحبوب العلفية ذات الطابع الاقتصادي على مستوى العالم لاحتوائها على الألياف بنسب كبيرة ويعد محصولا ذا أهمية كبيرة في ليبيا بسبب ارتباطه المباشر بقطاع الثروة الحيوانية، ويتحمل الشعير الظروف البيئية المعاكسة والجفاف وملوحة مياه الري، ويزرع محصول الشعير في المنطقة الشرقية بليبيا تحت ظروف الزراعة البعلية وكذلك يزرع في المشاريع الزراعية حيث بلغت الإنتاجية 5طن /هكتار (الشريدي ،2010)، إن لمعدلات البذار وطريقة توزيع النباتات في وحدة المساحة- تأثيراً كبيراً في مكونات الإنتاج الثلاثة في المحاصيل الحقلية، حيث أشار (عمر، 2009) أن طول السنبلة قد زاد معنوياً بزيادة الكثافة النباتية 80- 160كجم هكتار، كما توصلت (منى اللافي، 2009) عند دراستها تأثير الكثافة النباتية على طول السنبلة في كل من منطقة البيضاء ومنطقة القبة بشرق ليبيا أن طول السنبلة قد زاد معنوياً بزيادة الكثافة النباتية من 45-75 كجم/هكتار، ولزيادة الإنتاج وتحسين نوعيته يجب زراعة أصناف ملائمة للمنطقة، واستعمل 
أحدث التقنية الزراعية كالأصناف المحسنة والمستنبطة محليا في محطة مصراتة للبحوث الزراعية وغيرها من المراكز البحثية في ليبيا، مع استخدام الكثافة النباتية المناسبة للنمو والإنتاجية مما يجعل الصنف قادرا على إعطاء أفضل إنتاجية، ومن مبررات وأهدف البحث أن محصول الشعير من أهم المحاصيل وأقدمها في برقة وهو مصدر أساسي للعلف وتربية الأغنام وأفضل محصول في الزراعة البعلية، ولكن حصل له تراجع في الإنتاجية وذلك بسبب تدني مستوى أداء العمليات الزراعية لهذا المحصول حيث اقتصرت العمليات الزراعية على الحرث والحصاد فقط وهذا بدوره أدى إلى تراجع الإنتاجية بوحدة المساحة، ويهدف هذا البحث إلى معرفة تأثير ثلاث كثافات نباتية على نمو وإنتاجية ثلاثة أصناف من محصول الشعير.

\section{2. مواد وطرق البحث}

نفذت بتربة حقلية بمنطقة بئر إرجام شرق سلوق خلال الموسم الزراعي 2019/2018 بهدف معرفة تأثير الكثافات Split- النباتية المناسبة على نمو وإنتاجية ثلاثة أصناف من محصول الشعير صممت التجربة بالقطع المنشقة لمرة واحدة plot في أربع مكررات ووزعت الأصناف (الريحان، صفيت 65، مبشر4) بالقطع الرئيسية في حين وزعت معدلات البذار (70، 100، 130) كجم/هكتار بالقطع الثانوية وكانت مساحة القطعة التجريبة 8م²، جميع العمليات الزراعية نفذت طبقاً للمتبع في المنطقة لإنتاج حصول الشعير تحت نظام الري التكميلي، وخواص التربة الكيميائية والفيزيائية لمنطقة الدراسة موضحة بالجدول رقم (1) .تمت الزراعة في منتصف شهر نوفمبر أما السماد النيتروجيني فقد أضيفت بمعدل 135 كجم ن. هـ على دفعتين نصف الكمية عند الزراعة والنصف المتبقي في مرحلة الإشطاء في صورة يوريا 46 \% نيتروجين، تم قياس ارتفاع النبات (سم) من عشرة نباتات عشوائيا من الوحدة التجريبة من سطح التربة إلى قمة السنبلة

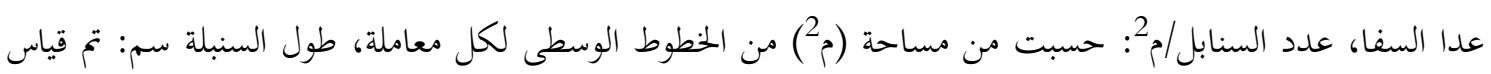
20 سنبلة بصورة عشوائية من وسط الوحدة التجريبية وأخذ المتوسط، عدد حبوب السنبلة: حسبت كمعدل لعدد الحبوب في عشرة سنابل مأخوذة عشوائيا من كل وحدة بتريبية، دليل الحبوب (وزن ال1000حبة): وزنت 1000 حبة مأخوذة بصورة عشوائية من حبوب السنابل المُصودة لكل وحدة تجريبية، ومن محصول القطعة التجريبية الكاملة تم حساب: محصول الحبوب (طن/هكتار)، تم تقدير محصول الحبوب على أساس وزن الحبوب (جم) للنباتات المصصودة من المساحة (8م²) من كل وحدة بحريبة، محتوى الحبوب من البروتين تم تقديره بمعمل شركة الجودة الفائقة لصناعات الدقيق ومشتقاته /سلوق، وذلك باستخدام جهاز . من شركة CHOPINeo Juniol. أجري التحليل الإحصائي للبيانات وفقط التصميم المتبع بمستوى معنوية 5\% وتمت المقارنة بين المتوسطات باختبار الأقل فرق معنوي (LSD). 
جدول (1) الخواص الفيزيائية والكيميائية للتربة منطقة الدراسة.

\begin{tabular}{|c|c|}
\hline 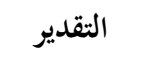 & الخاصية \\
\hline & الحواص الطبيعية \\
\hline 0.32 & الحصى (0\%) \\
\hline 28.16 & الرمل (\%\%) \\
\hline 42.43 & السلت (\%) \\
\hline 29.09 & الطين (\%) \\
\hline \multirow[t]{2}{*}{ طينية سلتيه } & 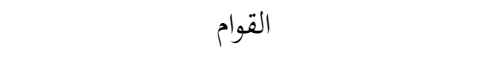 \\
\hline & الحواص الكيميائية \\
\hline 1.04 & المادة العضوية (\%) \\
\hline 19.01 & كربونات الكالسيوم (\%) \\
\hline 1.02 & التوصيل الكهربائي (مليموز/سم) عند 25 م \\
\hline 7.8 & الأس الهيدروجيني (pH) \\
\hline 0.7 & النيتروجين الكلي (\%) \\
\hline 7.8 & الفوسفور الميسر (جزء/مليون) \\
\hline
\end{tabular}

3. النتائج والمناقشة

1.3. ارتفاع النبات

البيانات الموضحة بجدول (2) تشير إلى وجود اختلافات معنوية بين الأصناف تحت الدراسة حيث تفوق صنف المبشر4 عن جميع الأصناف بصفة ارتفاع النبات وأعطى ارتفاع 76.33 سم، بينما كان أقل ارتفاع للنبات عند الصنف الريهان 68.22سم ويعزى ذلك إلى اختلاف معدل الانقسام بين الأصناف وبذلك أدت إلى اختلاف ارتفاعها، وهذه النتائج متفقه مع نتائج . حيث وجد أن أصناف الشعير تختلف في الارتفاع. من نفس الجدول تبين وجود فروق عالية المعنوية بين الكثافات النباتية حيث زاد ارتفاع النبات بزيادة الكثافة النباتية وأعطت الكثافة العالية أعلى ارتفاع للنبات75.78 سم بينما أقل ارتفاع عند الكثافة النباتية 70 كجم /هكتار بقيم67.89 سم، وتعزى الزيادة في ارتفاع النبات إلى زيادة الكثافة النباتية التي أثرت على النباتات بفعل التظليل ونقص الاشعاع الضوئي والذي أدى إلى دفع النباتات إلى سرعة الانقسام وزيادة الاستطالة للحصول على الضوء ويتفق هذا مع ما وجــــــ (Emam \& moaied,2000)، كان للتداخل بين الأصناف والكثافات النباتية تأثير معنوي الجلدول رقم (3) حيث أعطى الصنف مبشر4 عند معدل بذار 130كجم/هكتار أعلى ارتفاع للنبات (82.67). بينما أقل ارتفاع

$$
\text { عند صنف الريحان (65.67 سم). }
$$


جدول (2) تأثير الأصناف والكثافة النباتية والتداخل بينهما في ارتفاع النبات/ سم

\begin{tabular}{|c|c|c|}
\hline ارتفاع النبات & \multicolumn{2}{|c|}{ المعاملات } \\
\hline 68.22 & الريحان & \multirow{5}{*}{$\begin{array}{c}\text { الأصناف } \\
\text { (A) }\end{array}$} \\
\hline 71.00 & صفيت 65 & \\
\hline 76.33 & مبشر4 & \\
\hline * & F اختبار F & \\
\hline 5.340 & LSD & \\
\hline 67.89 & 70 & \\
\hline 71.89 & 100 & الكثافة النباتية \\
\hline 75.78 & 130 & \\
\hline *** & F اختبار F & (B) \\
\hline 2.147 & LSD & \\
\hline *** & $A * B$ & التفاعل \\
\hline
\end{tabular}

جدول (3) تأثير التداخل بين الأصناف والكثافة النباتية في ارتفاع النبات سم

\begin{tabular}{|c|c|c|c|}
\hline \multicolumn{3}{|c|}{ الكثافة النباتية } & \multirow{2}{*}{ الأصناف } \\
\hline 130 & 100 & 70 & \\
\hline 71.33 & 67.67 & 65.67 & الريحان \\
\hline 73.33 & 70.33 & 69.33 & صفيت 65 \\
\hline 82.67 & 77.67 & 68.67 & مبشر4 \\
\hline \multicolumn{3}{|c|}{5.420} & LSD \\
\hline
\end{tabular}

تشير النتائج الموضحة بجدول (4) إلى وجود تفوق عالي المعنوية بين الأصناف المدروسة بصفة عدد السنابل /P2 حيث أعطى الصنف صفيت 65 أعلى معدل من السنابل 308.8 سنبلة /م² بينما أقل عدد من السنابل نتجت عند صنف الريحان 277.1 سنبلة ويعزى ذلك إلى أن الأصناف ذات القابلية العالية من التفرع تعطي أعلى سنابل مقارنة بالأصناف القليلة التفرع واتفقت هذه النتائج مع (Zarina, 2014) الذي وجد اختلافا في عدد السنابل المتكونة في الأصناف، نلاحظ من البيانات في الجدول(4) تفوق الكثافة 130 كجم/ هـ بأعلى عدد من السنابل حيث أعطت 309.2سنبلة امُ2، في حين أن أقل معدل من السنابل حصلنا عليه عند الكثافة المنخفضة 70كجم/ه، ويعزى ذلك إلى زيادة الكثافة 
النباتية التي أدت إلى زيادة عدد الإشطاء في وحدة المساحة والتي انعكست على عدد السنابل المتكونة وتتفق هذه النتائج مع (Ramadhan,2013) حيث وجد أن زيادة عدد السنابل مرتبط بزيادة الكثافة النباتية في وحدة المساحة.

جدول (4) تأثير الأصناف والكثافة النباتية والتداخل بينهما في صفة عدد السنابل /م²

\begin{tabular}{|c|c|c|}
\hline عدد السنابل/م² & \multicolumn{2}{|c|}{ المعاملات } \\
\hline 277.1 & الريحان & \multirow{5}{*}{$\begin{array}{c}\text { الأصناف } \\
\text { (A) }\end{array}$} \\
\hline 308.8 & صفيت 65 & \\
\hline 263.3 & مبشر4 & \\
\hline *** & F اختبار F & \\
\hline 24.31 & LSD & \\
\hline 251.6 & 70 & \\
\hline 288.4 & 100 & الكثافة النباتية \\
\hline 309.2 & 130 & \\
\hline * & F اختبار F & ( B ) \\
\hline 20.75 & LSD & \\
\hline * & $A * B$ & التفاعل \\
\hline
\end{tabular}

كان للتداخل بين الأصناف والكثافة النباتية جدول (5) تأثير معنوي على صفة عدد السنابل /م² حيث تفوق الصنف الريحان عند الكثافة النباتية 130كجم/هـ بالحصول على أعلى معدل من السنابل 330سنبلة /م²، في حين أن أقل سنابل تم الحصول عليها عند زراعة الصنف مبشر 4 بالكثافة 70 كجم/هـ.

جدول (5) ثأثير التداخل بين الأصناف والكثافة النباتية في صفة عدد السنابل / م²

\begin{tabular}{|c|c|c|c|}
\hline \multicolumn{3}{|c|}{ الكثافة النباتية } & \multirow{2}{*}{ الأصناف } \\
\hline 130 & 100 & 70 & \\
\hline 330.0 & 260.3 & 241.0 & ريحان \\
\hline 305.3 & 340.7 & 280.3 & صفيت 65 \\
\hline 292.3 & 264.3 & 233.3 & مبشر4 \\
\hline \multicolumn{3}{|c|}{34.09} & LSD \\
\hline
\end{tabular}




\section{3. عدد الحبوب في السنبلة}

البيانات المعروضة بجدول (6) تشير إلى وجود فروق معنوية بين الأصناف والكثافة النباتية على صفة عدد الحبوب في السنبلة، حيث تفوق الصنف صفيت 65 على جميع الأصناف بصفة عدد حبوب السنبلة بعدد 40حبة/سنبلة بينما أقل عدد من الحبوب المتكون عند الصنف الريحان 36.67حبة/سنبلة، ويعزى الاختلاف إلى الخصائص الجينية للصنف، اتفقت هذه النتائج مع نتائج باحثين آخرين حيث توصلوا إلى أن الأصناف تختلف فيما بينها بصفة عدد الحبوب المتكونة بالسنبلة(Shafi et al., 2011) \&. أثرت الكثافة النباتية تأثير عالي المعنوي بصفة عدد الحبوب بالسنبلة حيث أدت زيادة الكثافة النباتية إلى حدوث نقص معنوي في عدد الحبوب بالسنبلة حيث أعطت الكثافة 100كجم /هـ أعلى عد من الحبوب المتكونة بالسنبلة (39.89) حبة/سنبلة مقارنة بالكثافة العالية التي أعطت أقل عدد من الحبوب بالسنبلة (36.78) حبة/سنبلة، ويعود هذا إلى شدة التنافس البيني في الكثافات العالية والذي بدوره أثر على مواقع نشوء الحبوب بالسنبلة فينخفض عدد الحبوب في كل سنبلة ويحدد هذا الانخفاض بقابلية النبات على التنافس مع النباتات الأخرى، ويؤكد ذلك كل من.(Subhan \& Jamro,2003 ; Iqbal et al., 2010) الذين وجدوا أن زيادة الكثافة النباتية قد أدت إلى حدوث انخفاض معنوي في متوسط عدد الحبوب بالسنبلة.

جدول (6) تأثير الأصناف والكثافة النباتية في صفة عدد حبوب السنبلة

\begin{tabular}{|c|c|c|}
\hline عدد حبوب السنبلة & \multicolumn{2}{|c|}{ المعاملات } \\
\hline 36.67 & الريحان & \multirow{5}{*}{ الأصناف } \\
\hline 40.00 & صفيت 65 & \\
\hline 37.67 & ل & \\
\hline * & Fتبار F اخت & \\
\hline 1.926 & LSD & \\
\hline 37.67 & 70 & \multirow{5}{*}{ لكثافة النباتية } \\
\hline 39.89 & 100 & \\
\hline 36.78 & 130 & \\
\hline *** & F اختبار F & \\
\hline 1.937 & LSD & \\
\hline N.S & $A * B$ & التفاعل \\
\hline
\end{tabular}


يشير جدول (7) إلى فروق معنوية بين الأصناف والكثافات النباتية والتداخل بينهما على صفة دليل الحبوب حيث تفوق الصنف صفيت 65 على جميع الأصناف، حيث أعطى أعلى متوسط لوزن ألف حبة بلغ 38.11جم بينما أعطى الصنفان ريحان ومبشر4 أقل متوسط لمذه الصفة بلغا 33.33 و 33.32جم على التوالي، يعزى السبب إلى الاختلافات

الوراثية بين الأصناف، وقد توافقت النتاج مع ما توصل إليه (Khalil et al., 2006). أثرت الكثافة النباتية تأثيراً عالي المعنوية على صفة 1000حبة حيث أعطت الكثافة المنخفضة 70كجم/هـ أعلى وزن حيث بلغت 37.38جم في حين أقل وزن سجل عند الكثافة العالية 130كجم/هـ بـ32.56 جم يعزى سبب الانخفاض عند الكثافة العالية إلى قلة ترسب المادة الجافة في الحبوب بسبب المنافسة الشديدة على الماء والعناصر الغذائية بين النباتات، واتفقت هذه النتائج مع نتائج كل من (Soomro et al.,2009; Malik.et al.,2009). حيث وجدوا أن وزن حبوب السنبلة ينخفض بزيادة الكثافات النباتية.

جدول (7) تأثير الأصناف والكثافة النباتية والتداخل بينهما في صفة وزن 1000حبة

\begin{tabular}{|c|c|c|}
\hline دليل الحبوب وزن 1000 حبة & \multicolumn{2}{|c|}{ المعاملات } \\
\hline 33.33 & الريحان & \multirow{5}{*}{$\begin{array}{c}\text { الأصناف } \\
\text { (A) }\end{array}$} \\
\hline 38.11 & صفيت 65 & \\
\hline 33.22 & مبشر4 & \\
\hline *** & F اختبار F & \\
\hline 0.976 & LSD & \\
\hline 37.78 & 70 & \multirow{6}{*}{ الكثافة النباتية } \\
\hline 34.33 & 100 & \\
\hline 32.56 & 130 & \\
\hline *** & F اختبار F & \\
\hline 1.118 & LSD & \\
\hline * & $A * B$ & \\
\hline
\end{tabular}

أثنَّ التداخل ببن الأصناف والكثافة النباتية تأثيراً معنوياً عاليا على صفة وزن ال1000 حبة حيث أعطى الصنف صفيت 65 أعلى وزن للحبوب عند الكثافة النباتية المنخفضة 70 كجم/هـ بلغ 42.33جم، في حين أقل وزن سجله عند الصنف مبشر4 عند الكثافة العالية ب31.00 جم. 
جدول (8) تأثير الأصناف والكثافة النباتية والتداخل بينهما في صفة وزن 1000حبة

\begin{tabular}{|c|c|c|c|}
\hline \multicolumn{3}{|c|}{ الكثافة النباتية } & \multirow{2}{*}{ الأصناف } \\
\hline 130 & 100 & 75 & \\
\hline 31.33 & 33.67 & 35.00 & الريحان \\
\hline 35.33 & 36.67 & 42.33 & صفيت 65 \\
\hline 31.00 & 32.67 & 36.0 & مبشر4 \\
\hline \multicolumn{3}{|c|}{1.712} & LSD \\
\hline
\end{tabular}

5.3.

البيانات الموضحة بجدول (9) تشير إلى وجود تأثير معنوي بين الأصناف والكثافات النباتية والتفاعل بينهما على صفة محصول الحبوب حيث تفوق الصنف صفيت 65 تفوقاً عالي المعنوية على جميع الأصناف تحت الدراسة فقد أعطى أعلى وزن لمصول الحبوب بـ3.289 طن/ هكتار حبوب بينما أقل محصول سجله عند الصنف الريحان بوزن 2.423 طن/هكتار، يعزى اختلاف الأصناف المدروسة في محصول الحبوب إلى اختلافها في التركيب الوراثي، والذي انعكس بدوره علي التأثير في مكونات المحصول المختلفة، حيث تفوق الصنف صفيت 65في صفة عدد السنابل/م² جدول (4) ، ووزن الا1000حبة جدول (7)، وقد توصل إلى نتائج مشابهة الباحث (Yoshihira et al.,2002) الذي وجد أن سبب الزيادة يرجع إلى اختلاف الأصناف فيما بينها في ححصول الحبوب الكلي. أظهرت نتائج الجدول (9) أن التغير في الكثافة النباتية قد أثر معنوياً على محصول الحبوب بوحدة المساحة حيث زاد المحصول تدريجياً بزيادة الكثافة النباتية من 75- 130كجم/هكتار في موسم الزراعة حيث أعطت الكثافة العالية ( 130 كجم/هكتار) أعلى محصول 3.156 طن/هكتار، مقارنة بالمصول الناتج عن الزراعة بمعدل 70كجم/هكتار حيث بلغ إنتاجه 2.548طن/هكتار، وقد يعزى ذلك إلى زيادة واحد أو أكثر من مكونات المصصول مثل عدد السنابل/م² جدول (4) عدد حبوب السنبلة جدول (6) ووزن ال1000حبة وتتفق هذه النتائج مع نتائج (O'Denovan et al. 2011). 
جدول (9) تأثير الأصناف والكثافة النباتية والتداخل بينهما في صفة محصول الحبوب

\begin{tabular}{|c|c|c|}
\hline محصول الحبوب طن/هكتار & & \\
\hline 2.423 & ريحان & \multirow{5}{*}{$\begin{array}{l}\text { الأصناف } \\
\text { (A) }\end{array}$} \\
\hline 3.289 & صفيت 65 & \\
\hline 3.002 & | & \\
\hline *** & F اختبار F & \\
\hline 0.2070 & LSD & \\
\hline 2.548 & 70 & \multirow{5}{*}{ الكثافة النباتية } \\
\hline 3.011 & 100 & \\
\hline 3.156 & 130 & \\
\hline 㐘 & F اختبار F & \\
\hline 0.1553 & LSD & \\
\hline N.S & $A * B$ & التفاعل \\
\hline
\end{tabular}

6.3. محتوى الحبوب من البروتين (\%)

يتبين من الجدول (10) تفوق الصنف مبشر 4 معنويا على صنفي الريحان وصفيت 65 بإعطاء أعلى نسبة بروتين (8.156 \%)، وسجل الصنف الريحان أقل نسبة بروتين (6.856 \%) ويعزى اختلاف الأصناف في نسبة البروتين إلى اختلافها في تركيبها الوراثي، وهذا يتوافق مع ما أشار إليه (Kamal et al., 2003) بأن الأصناف تختلف في محتوى

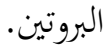
جدول (10) تأثير الأصناف والكثافة النباتية والتداخل بينهما في محتوي الحبوب من البروتين

\begin{tabular}{|c|c|c|}
\hline محتوي الحبوب من البروتين (\%) & 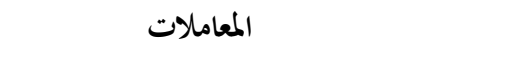 & \multirow{6}{*}{$\begin{array}{c}\text { الأصناف } \\
\text { (A) }\end{array}$} \\
\hline 6.856 & 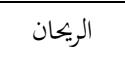 & \\
\hline 7.611 & صفيت 65 & \\
\hline 8.156 & 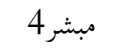 & \\
\hline * & 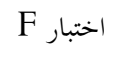 & \\
\hline 0.2953 & LSD & \\
\hline 7.233 & 70 & \\
\hline 7.478 & 100 & \\
\hline 7.911 & 130 & الكثافة النباتية \\
\hline *** & 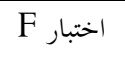 & ( B ) \\
\hline 0.2138 & LSD & \\
\hline N.S & A*B & التفاعل \\
\hline
\end{tabular}


تشير البيانات الموضحة بالجدول (10) إلى وجود تأثير عالي المعنوية لمعدلات البذار في محتوي الحبوب من البروتين حيث زادت نسبة البروتين بزيادة معدلات البذار 70 إلى 130 كجم/ هكتار في الزراعة بمعدل 130كجم/هكتار أعطت أعلى محتوى من البروتين بـ7.911\% بينما الزراعة بمعدل 70 كجم/هكتار أعطت 7.233 \%، وتتفق هذه النتائج مع ما توصل إليه (Kwabiah, 2005)، ولم يكن لعامل التداخل بين الأصناف والكثافة النباتية أي تأثير معنوي على محتوى الحبوب من البروتين.

\section{الخلاصة:}

خلصت الدراسة إلى أن أداء الأصناف اختلف فيما بينها وفق التركيب الوراثي والتأقلم مع الظروف البيئية الخاصة بالمنطقة حيث تفوق صنف صفيت 65 في صفة إنتاجية الحبوب وتفوق صنف مبشر 4 في المختوى من البروتين، وكانت الكثافة النباتية 100 كلجم/هكتار هي الأنسب لزراعة الشعير بالمنطقة.

الشريدي ع. س. (2010)، وضع محصول الشعير في ليبيا، دراسة مرجعية حول محصولي القمح والشعير في ليبيا، مركز البحوث الزراعية ليبيا

اللافف .م. ع (2009). استجابة بعض التراكيب الوراثية للشعير لمعدلات البذار المختلفة تحت ظروف الزراعة المطرية بمنطقة الجبل الأخضر. رسالة ماجستير، كلية الزراعة، جامعة عمر المختار، ليبيا.

عمر .ر. ش (2009). تأثير الكثافة النباتية ومستوى التسميد النتروجينى وميعاد الحش على نمو ومكونات مصصول الشعير تحت ظروف الجبل الأخضر. رسالة ماجستير، كلية الزراعة، جامعة عمر المختار، ليبيا.

Emam, Y, \& Moaied, G.R. (2000). Effect of planting density and chlormequat chloride on morphological and physiological characteristics of winter barley (Hordeum vulgare L.) cultivar Valfajr. Journal of Agriculture Science and Technology, 2, 75-83

Iqbal, N., N. Akbar, M. Ali, M. sattar \& L. Ali. ( 2010). Effect of seed rate and row spacing on yield components of wheat Triticum aestivum L. J. Agric 48(2):151-156.

Kamal, A. M.A.; M.R. Islam; B.L.D. Chowdhry \& M.A. MalequeTalukder .(2003). Yield performance and grain quality of wheat varieties grown under rainfed and irrigated conditions. Asian J. of plant Sci. 2: 358 - 360 
Khalil,F.A.F.; A.M. Tammam; I.A. Amin \& K.A. Mohamed .(2006).Scheduling irrigation for some wheat cultivars under upper Egypt conditions. J. Agric. Sci. Mansoura Univ. 31:561-572.

KwabiahA.B.(2005). Forage and nitrogen yield of barley as influenced by seeding and N rates .J. of Noew Seeds,7(1):43-51.

Malik, A. U., M. Ahmad, H.A. Bukhsh \& I. Hussain .(2009). Effect of seed rates on different. Dates of wheat under agro-ecological condition of deraghazikhan .

J. Animal \& plant. Sci. 19(3): 126-129.

Mousavi, M., A. Soleyman \& M. Shams.( 2012). Changes in yield and yield components of three cultivar, of barley under different nitrogen levels in Isfahan region. Inter, J. Agric. Crop Sci. Vol. 4(19): 1433-1435.

O'Denovan, J. T.; T. K. Turkington; M. J. Edney \& G. W. Clayton.( 2011). Seedling rate, nitrogen rate and cultivars effect on malting Barley Production. Agron. J. 103(3): 709-716.

Ramadhan, M. N. (2013). Tillage systems and seeding rate effect on yield components, seed yield \& biological yield of barley cultivars. J. of Basrah Res. 39(1): 33-46.

Shafi, M.; J. Bakht; F. Jalal; M. Amankhan and S.G Khattak..( 2011). Effect of nitrogen application on yield and yield components of barley (HordeumVulgarel.). Pak. J. Bot., 43(3): 1471- 1475.

Soomro, U. A., M. Ur Rahman, E. A. Odhano, S.Gul \& A. Q. Tareen .(2009). Effects of sowing method and seed rate on growth and yield of wheat Triticum aestivum. World J. of Agric. Sci. 5(2) 159-162.

Subhan, F.,M.Khan \& G.H.Jamro.(2003). Weed management through planting date, seeding rate and weed control method in wheat. Pak.J. weed Sci. Res. 9(1-2):49-57.

Yoshihira, T.; T. Karasawa and K. Nakatsuka (2002). Traits associated with high-yield in winter triticale in Hokkaido, Japan comparison with wheat and rye. A. Growth analysis. Proceedings of the 5th International Triticale Symposium, Radzikow, Poland, 30 June - 5 July, Volume II: poster presentations; 2002. 141-148.

Zarina, Y., N.Paltridge, R. Graham,B.Huynh, \& J.Stangoulis.(2014). Measuring genotypic variation in wheat seed iron first requires stringent protocols to minimize soil iron contamination. Crop Sci., 54(1): 255-264. 


\title{
The effect of plant density on the growth and yield of three cultivars of barley
}

\author{
Abdul Halim Rajab Indoush*
}

Department of Crops, Faculty of Agriculture, University of Benghazi

*abdulhalim.inooush@uob.edu.ly

\begin{abstract}
:
A field experiment was carried out in Bir Arjam area east of Suluq during the agricultural season 2018/2019 in order to know the effect of plant densities on the growth and yield of three cultivars of barley crop. The study was carried out with the design of split plot with four replicates in which cultivars (Rihan, Safet-65, Mebsher-4), were assigned to main plots and Seeding rates $(70,100,130) \mathrm{kg}$ seeds/ha to sub plots. The experimental unit area was $8 \mathrm{~m}^{2}$. All agricultural operations were carried out according to the regulations used in the region to produce the barley crop. Planting took place in mid-November. As for nitrogen fertilizer, it was added at a rate of $135 \mathrm{~kg} \mathrm{~N} / \mathrm{ha}$ in two batches, half the amount when planting and the remaining half at the branching stage. The increase in plant density led to an increase in plant height, the higher density gave the highest height $75.78 \mathrm{~cm}$, and the highest cereal yield was $3.156 \mathrm{t} / \mathrm{ha}$. The highest protein content was $7.911 \%$. While the cultivar (Safet-65) was given the highest number of spikes 308.8 spike/m2 and the highest weight of cereal yield was 3.289 tons/hectare, whereas the highest protein content in cereals for the variety Mebsher-4 was $8.156 \%$.
\end{abstract}

Keywords: Cultivars - Barley - Plant density - Growth - Productivity - Suluq. 